'Servicio de Nefrología, Hospital Las Higueras de Talcahuano. Talcahuano, Chile. ${ }^{2}$ Departamento de Medicina Interna, Facultad de Medicina, Universidad de Concepción. Concepción, Chile.

${ }^{3}$ Departamento de Salud

Pública, Facultad de Medicina, Universidad de Concepción. Concepción, Chile. ${ }^{4}$ Departamento de Estadística, Facultad de Ciencias Físicas y Matemáticas, Universidad de Concepción. Concepción, Chile. ${ }^{5}$ Unidad de Paciente Crítico Adulto, Hospital Las Higueras de Talcahuano. Talcahuano, Chile. ${ }^{6}$ Instituto de Nefrología Concepción. Concepción, Chile. apsicólogo. bMatrón.

'Profesora de Matemática y Computación. ${ }^{\mathrm{d}}$ Enfermera.

eCandidato a Doctor en Epidemiología y Salud Pública por la Universidad Autónoma de Madrid, España.

Este estudio no recibió financiamiento específico. Los autores declaran no tener conflictos de interés.

Recibido el 24 de octubre de 2018, aceptado el 12 de abril de 2019.

Correspondencia a: Dr. Hans Müller Ortiz, FACP Hospital Las Higueras de Talcahuano, Calle Alto Horno 777, Las Higueras, Talcahuano. hmuller@me.com

\section{Prevalencias de complicaciones asociadas a la instalación de catéter venoso central para hemodiálisis}

\author{
HANS MÜLLER-ORTIZ ${ }^{1,2,6}$, CRISTIAN PEDREROS-ROSALES ${ }^{1,2,5,6}$, \\ JUAN PABLO SILVA-CARVAJAL ${ }^{1,5}$, DAVID KRAUNIK-RODRÍGUEZ ${ }^{1,2,5}$, \\ ALDO VERA-CALZARETTA ${ }^{3, \mathrm{a,f},}$, ALEXIS GONZÁLEZ-BURBOA ${ }^{3, \mathrm{~b}, \mathrm{e},}$, RENÉ \\ CAMENT-MUÑOZ2, SUSAN TAYLOR-MONTOYA2, \\ NATALIA MÜLLER-HENRÍQUEZ ${ }^{2}$, TATIANA CHÁVEZ-CAMPODÓNICO ${ }^{1, \mathrm{~d}}$, \\ LUISA RIVAS-CALABRÁN ${ }^{4, c, f}$
}

\section{Prevalence of complications associated with central venous catheter instalation for hemodyalisis}

Background: Placing central venous lines under ultrasonographic guidance reduces the complications of the procedure. Aim: To compare prevalences of complications of central venous line placements with or without ultrasonographic guidance. Material and Methods: Descriptive study that contemplated the comparison of two groups of patients subjected to a central venous line placement at a nephrology service for renal replacement therapy. In one group of 100 patients, the line was placed without ultrasonographic guidance between 2008 and 2012. Between 2015 and 2017 the line was placed in 138 patients using ultrasonographic guidance. The prevalences of complications with both types of procedures were recorded. Results: The frequency of complications of procedures with and without ultrasonographic guidance was 0.7 and $18 \%$ respectively (prevalence ratio 0.04 , 95\% confidence interval 0-0.3). Ninety five percent of recorded complications were arterial puncture, followed by hematomas in 10\% and pneumothorax in 5\%. The higher prevalence of complications was observed in emergency line placement without ultrasonographic guidance. There was a direct association between the number of line placement attempts in a single procedure and the prevalence of complications. Conclusions: Ultrasonographic guidance is associated with a reduction in the prevalence of central venous line complications.

(Rev Med Chile 2019; 147: 458-464)

Key words: Central Venous Catheters; Dialysis; Renal Replacement Therapy; Ultrasonography.
E 1 uso de una vía venosa central es muy frecuente en la práctica clínica, siendo empleada en monitoreo hemodinámico, administración de hemoderivados y fármacos, nutrición parenteral, fluidoterapia y terapias de reemplazo renal ${ }^{1}$. Por lo anterior, se considera que la instalación del catéter venoso central (CVC) es una competencia clínica relevante, cuyo éxito depende significativamente de la anatomía del paciente, las comorbilidades de este y la habilidad del operador ${ }^{2}$.

Desde el desarrollo de la técnica percutánea por el radiólogo Sven-Ivar Seldinger en 1952, el uso de catéteres centrales se amplió a diversas 
áreas de la medicina. Tradicionalmente, el sitio de punción de la aguja se determina mediante el uso de referencias anatómicas palpables o visibles, relacionadas con la vena que se desea abordar ${ }^{3}$.

Esta técnica, ampliamente utilizada, tiene complicaciones asociadas. La literatura señala que la frecuencia de complicaciones graves podría superar $20 \%{ }^{4}$, siendo las más relevantes la punción arterial, el neumotórax, el hemotórax, la punción del plexo braquial y la mala posición del $\mathrm{CVC}^{5}$. La frecuencia y consecuencias de dichas complicaciones están condicionadas por la anatomía de los pacientes. Niños, personas obesas y aquellos con cuello más corto tienen un abordaje más complejo. Además, las variantes anatómicas normales y la trombosis de la vena pueden hacer que aun el operador experto introduzca la aguja en una dirección inapropiada. Por otra parte, pacientes con trastornos de la coagulación, en ventilación mecánica y aquellos que requieren instalación de marcapaso de urgencia, tienen más riesgo de complicación asociada a la venopunción ${ }^{6}$. Por último, los pacientes que requieren $\mathrm{CVC}$ de forma repetida (quimioterapia o hemodiálisis), poseen significativamente más riesgo de formación de trombos ${ }^{1}$.

Dadas las limitaciones previamente descritas, los reportes del uso de ultrasonografía (US) como apoyo en la canulación de una vía venosa central han aumentado considerablemente desde la década de los noventa. El desarrollo de esta técnica ha permitido una reducción importante en las complicaciones asociadas a la punción venosa y un aumento de la frecuencia de canulación en "un solo intento" de la vena yugular interna ${ }^{7}$, existiendo menos evidencia en vena femoral, vena subclavia y en pacientes pediátricos ${ }^{8}$.

Hoy en día, la guía ultrasonográfica ha aumentado el éxito y la seguridad de una miríada de procedimientos invasivos, tales como toracocentesis, paracentesis, artrocentesis, anestesia regional, drenaje de abscesos, localización y remoción de cuerpos extraños, punción lumbar, biopsias, etc. ${ }^{9}$. Existen dos formas de apoyo por US: apoyo estático y dinámico. Con la guía estática la estructura es identificada y se marca el sitio y ángulo de entrada en la piel. En procedimientos dinámicos, la US visualiza la aguja en "tiempo real" durante la punción. Si bien esta última forma requiere algo más de entrenamiento, brinda sin duda más apoyo durante cualquier procedimiento ${ }^{1}$. Por esto el uso de guía ultrasonográfica dinámica durante la instalación de un CVC ha sido considerada una de las doce prácticas de seguridad mejor valoradas y que contribuye a disminuir los errores médicos ${ }^{10,11}$.

Desafortunadamente, la incorporación de estas recomendaciones dentro de la práctica clínica habitual ha encontrado resistencia por parte de los médicos. Se ha reportado que más del $40 \%$ de los operadores no usa el apoyo ultrasonográfico pese a disponer del recurso, ya que consideran tener un entrenamiento inadecuado o piensan que su experiencia en el uso de referencias anatómicas les otorga menor probabilidad de error ${ }^{7,12}$.

Creemos que en nuestro país existe un número importante de equipos de ultrasonido disponibles, ya sea en unidades de paciente crítico u otras dependencias, que facilitarían la instalación de CVC. Sin embargo, no existen reportes locales acerca de la implementación de esta técnica y de sus resultados.

El objetivo principal de este estudio fue comparar la prevalencia de complicaciones asociadas a la instalación de una vía venosa central mediante el uso de guía ultrasonográfica, con la prevalencia de complicaciones ocurridas al utilizar el método convencional basado en referencias anatómicas, en pacientes del Hospital Las Higueras de Talcahuano que requirieron terapia de sustitución renal, administración de fármacos o monitoreo hemodinámico. En forma secundaria se buscó caracterizar las complicaciones de ambos grupos en relación con la programación del procedimiento, sitio de punción, tipo de complicación y número de punciones realizadas.

\section{Material y Método}

\section{Diseño}

El diseño del estudio fue descriptivo y contempló la comparación de dos cortes transversales.

\section{Población}

La población quedó constituida por personas de 18 o más años que fueron sometidas a la instalación de un CVC en el Servicio de Nefrología del Hospital Las Higueras del Servicio de Salud Talcahuano, Chile, entre los años 2008 y 2017.

Para la instalación de la vía referida se utilizó uno de los siguientes métodos: (a) basado en los puntos de referencia anatómica (convencional) 
y (b) con guía ultrasonográfica. Al respecto, la instalación en el grupo convencional fue realizada por cuatro médicos nefrólogos y un médico internista, todos entrenados en la técnica y con una experiencia previa de más de 300 casos cada uno. Por otra parte, en el grupo con guía ultrasonográfica, el procedimiento fue llevado a cabo por dos médicos nefrólogos y un médico internista con entrenamiento certificado en la técnica, cada uno con una curva de aprendizaje previa (tutorizada) de 30 casos. Todos los médicos pertenecían al Servicio de Nefrología.

\section{Sujetos}

Los datos fueron obtenidos desde los registros de las bases de datos anónimas del Servicio de $\mathrm{Ne}$ frología y Diálisis del Hospital Las Higueras. Estos registros, por solicitud del Comité Ético Científico evaluador, fueron anonimizados por un tercero, responsable de la gestión de las bases de datos en el servicio clínico, que también proveyó dichas bases al investigador responsable del estudio, quien finalmente resguardó y administró los datos en lo que respecta a su procesamiento y análisis.

El resto del equipo de investigación solamente accedió a los datos en la medida en que esto fue facilitado por el investigador responsable.

\section{Criterios de inclusión y exclusión a la muestra}

Como criterios de inclusión se consideraron: (a) tener una edad igual o mayor a 18 años; (b) contar con una indicación médica de instalación de vía venosa central y (c) tener pruebas de coagulación normales (INR menor o igual a 1,2; TTPK $<40$ segundos; Plaquetas $>100.000 \mathrm{~mm}^{3}$ ). Fueron excluidas del estudio aquellas personas que no poseían un registro completo de los datos del procedimiento (número de punciones, complicaciones, control radiográfico, etc.).

Cabe señalar que todas las personas incluidas en este estudio cumplieron con la condición de haber sido sometidas a la instalación de un catéter venoso central, mediante el procedimiento basado en los puntos de referencia anatómica (estándar) o guiado por US, por única vez.

\section{Constitución de los grupos}

a) Grupo 1 (Primer corte transversal)

Este grupo quedó constituido por pacientes que fueron sometidos a la instalación de un catéter venoso central, con la utilización del método basado en los puntos de referencia anatómica (estándar) entre los años 2008 y 2012.

Para la selección de los sujetos se utilizó un muestreo aleatorio simple. El tamaño de la muestra fue calculado utilizando la fórmula de construcción de intervalos de confianza para proporciones con población conocida (360 casos), considerando un nivel de confianza de $95 \%$ y un error de $5 \%$. Además, para el cálculo se consideró 8,5\% aproximado de prevalencia esperada de complicaciones en la instalación de $\mathrm{CVC}^{13}$. Dado lo anterior, se estimó un tamaño muestral mínimo requerido de 92 sujetos, pero esta cifra se incrementó en $10 \%$ contemplando una eventual falta de información en las fichas de registro, quedando constituida la muestra por 100 sujetos.

\section{b) Grupo 2 (Segundo corte transversal)}

El grupo quedó constituido por aquellos pacientes en los cuales la instalación del CVC fue con guía ultrasonográfica dinámica. En este caso se trabajó con la totalidad de la población atendida entre los años 2015 y 2017, cuyo tamaño fue de 138.

Para el período comprendido entre los años 2013 y 2014 no se seleccionaron individuos, debido a que quienes realizaban la intervención se encontraban en proceso de capacitación.

\section{Análisis estadístico}

Se llevaron a cabo análisis descriptivos univariados y bivariados, utilizando tablas de frecuencias, proporciones, medidas de tendencia central y de dispersión.

A partir de las prevalencias obtenidas para cada grupo, como medida de asociación, se estimó la razón de prevalencias.

Los datos fueron procesados utilizando los paquetes estadísticos STATA 14 y Epidat Versión 3.1.

El presente proyecto contó con la aprobación del Comité Ético Científico del Servicio de Salud Talcahuano.

\section{Resultados}

El promedio de edad de los sujetos fue de 59,7 $(\mathrm{DE}=14,6)$ años (Tabla 1). Un 54\% fueron mujeres con una edad promedio de 58,7 $(\mathrm{DE}=14,6)$ años. En el caso de los hombres, el promedio de edad fue de 60,8 (DE =14,5 años). 
Tabla 1. Tipos de complicaciones según procedimiento $(\mathbf{n}=\mathbf{2 3 7})$

\begin{tabular}{|c|c|c|c|}
\hline & Total & $\begin{array}{c}\text { Guía } \\
\text { ultrasonográfica }\end{array}$ & $\begin{array}{c}\text { Procedimiento } \\
\text { convencional }\end{array}$ \\
\hline \multicolumn{4}{|l|}{ Antecedentes sociodemográficos } \\
\hline Edad, promedio $\pm \mathrm{DE}$ & $59,7 \pm 14,6$ & $60,5 \pm 14,5$ & $58,7 \pm 14,7$ \\
\hline \multicolumn{4}{|l|}{ Sexo, $n(\%)$} \\
\hline Mujer & $128(54,0)$ & $69(50,4)$ & $59(59,0)$ \\
\hline Hombre & $109(46,0)$ & $68(49,6)$ & $41(41,0)$ \\
\hline \multicolumn{4}{|l|}{ Antecedentes del procedimiento } \\
\hline \multicolumn{4}{|l|}{ Programación del procedimiento, n (\%) } \\
\hline Electivo & $145(61,2)$ & $103(75,2)$ & $42(42,0)$ \\
\hline Urgencia & $92(38,8)$ & $34(24,8)$ & $58(58,0)$ \\
\hline \multicolumn{4}{|l|}{ Motivo de la intervención, n (\%) } \\
\hline Inicio terapia sustitución renal & $233(98,3)$ & $137(100)$ & $96(96,0)$ \\
\hline Otras & $4(1,7)$ & $0 \quad(0,0)$ & $4(4,0)$ \\
\hline \multicolumn{4}{|l|}{ Sitio de punción, n (\%) } \\
\hline Femoral & $23(9,7)$ & $9 \quad(6,6)$ & $14(14,0)$ \\
\hline Subclavio & $4(1,7)$ & $1 \quad(0,7)$ & $3(3,0)$ \\
\hline Yugular & $210(88,6)$ & $127(92,7)$ & $83(83,0)$ \\
\hline Número de punciones, promedio $\pm \mathrm{DE}$ & $1,2 \pm 0,5$ & $1,0 \pm 0,2$ & $1,4 \pm 0,7$ \\
\hline \multicolumn{4}{|l|}{ Complicaciones del procedimiento } \\
\hline \multicolumn{4}{|l|}{ Número de complicaciones, n (\%) } \\
\hline 0 & $218(92,0)$ & $136(99,3)$ & $82(82,0)$ \\
\hline 1 & $17(7,2)$ & $1 \quad(0,7)$ & $16(16,0)$ \\
\hline 2 & $2(0,8)$ & $0 \quad(0,0)$ & $2(2,0)$ \\
\hline \multicolumn{4}{|l|}{ Punción arterial, n (\%) } \\
\hline No & $219(92,4)$ & $136(99,3)$ & $83(83,0)$ \\
\hline $\mathrm{Si}$ & $18(7,6)$ & $1 \quad(0,7)$ & $17(17,0)$ \\
\hline \multicolumn{4}{|l|}{ Hematoma, n (\%) } \\
\hline No & $235(99,2)$ & $137(100)$ & $98(98,0)$ \\
\hline $\mathrm{Si}$ & $2(0,8)$ & $0 \quad(0,0)$ & $2(2,0)$ \\
\hline \multicolumn{4}{|l|}{ Neumotórax, n (\%) } \\
\hline No & $236(99,6)$ & $137(100)$ & $99(99,0)$ \\
\hline $\mathrm{Si}$ & $1(0,4)$ & $0 \quad(0,0)$ & $1(1,0)$ \\
\hline
\end{tabular}

Desde un punto de vista descriptivo, cabe señalar que del total de las vías venosas instaladas, $61,2 \%$ se realizó de forma electiva, siendo mayoritariamente por indicación de terapia de sustitución renal $(98,3 \%)$. El sitio de punción más utilizado fue la vena yugular $(88,6 \%)$, seguido por la vena femoral $(9,7 \%)$ y la vena subclavia $(1,7 \%)$. En cuanto al número de punciones realizadas, en promedio se realizaron $1,2(\mathrm{DE}=0,5)$ punciones para lograr una instalación exitosa.

En 218 procedimientos realizados, se conta- ron 19 complicaciones, lo que corresponde a una prevalencia de $8,7 \%$. Del total de intervenciones realizadas $7,2 \%$ tuvo una sola complicación y $0,8 \%$ tuvo dos. Del total de complicaciones, $94,7 \%$ correspondieron a punciones arteriales, $10,5 \%$ a hematomas en el sitio de punción y 5,2\% a neumotórax.

Respecto a la prevalencia de complicaciones en los grupos estudiados, $0,7 \%$ de los pacientes que fueron sometidos a US presentaron complicaciones. Para quienes recibieron el procedimiento 
Tabla 2. Aspectos relativos al procedimiento según técnica y presencia de complicaciones $(\mathbf{n}=237)$

\begin{tabular}{|c|c|c|c|c|c|c|c|}
\hline & \multirow[b]{2}{*}{ Total } & \multicolumn{3}{|c|}{ Guía ultrasonográfica } & \multicolumn{2}{|c|}{ Convencional } & \multirow[b]{2}{*}{ Total } \\
\hline & & SC & CC & Total & SC & CC & \\
\hline \multicolumn{8}{|l|}{ Programación, n (\%) } \\
\hline Electiva & $145(61,2)$ & $102(99,0)$ & $1(1,0)$ & $103(75,2)$ & $36(85,7)$ & $6(14,3)$ & $42(42,0)$ \\
\hline Urgencia & $92(38,8)$ & $34(100)$ & $0(0,0)$ & $34(24,8)$ & $46(79,3)$ & $12(20,7)$ & $58(58,0)$ \\
\hline \multicolumn{8}{|l|}{ Sitio de punción, n (\%) } \\
\hline Femoral & $23 \quad(9,7)$ & $9(100)$ & $0(0,0)$ & $9(6,6)$ & $8(57,1)$ & $6(42,9)$ & $14(14,0)$ \\
\hline Subclavia & $4(1,7)$ & $1(100)$ & $0(0,0)$ & $1 \quad(0,7)$ & $3(100)$ & $0 \quad(0,0)$ & $3(3,0)$ \\
\hline Yugular & $210(88,6)$ & $126(99,2)$ & $1(0,8)$ & $127(72,7)$ & $71(85,5)$ & $12(14,5)$ & $83(83,0)$ \\
\hline \multicolumn{8}{|l|}{ Motivo, n (\%) } \\
\hline Inicio terapia sustitución renal & $233(98,3)$ & $136(99,3)$ & $1(0,7)$ & $137(100)$ & $79(82,3)$ & $17(17,7)$ & $96(96,0)$ \\
\hline Otro $^{1}$ & $4(1,7)$ & $0 \quad(0,0)$ & $0(0,0)$ & $0 \quad(0,0)$ & $3(75,0)$ & $1(25,0)$ & $4(4,0)$ \\
\hline \multicolumn{8}{|l|}{ Número de punciones, n (\%) } \\
\hline 1 & $203(85,7)$ & $132(99,2)$ & $1(0,8)$ & $133(97,1)$ & $67(95,7)$ & $3(4,3)$ & $70(70,0)$ \\
\hline 2 & $25(10,5)$ & $4(100)$ & $0(0,0)$ & $4 \quad(2,9)$ & $14(66,7)$ & $7(33,3)$ & $21(21,0)$ \\
\hline 3 & $6(2,5)$ & $0 \quad(0,0)$ & $0(0,0)$ & $0 \quad(0,0)$ & $1(16,7)$ & $5(83,3)$ & $6(6,0)$ \\
\hline 4 & $3(1,3)$ & $0 \quad(0,0)$ & $0(0,0)$ & $0 \quad(0,0)$ & $0 \quad(0,0)$ & $3(100)$ & $3(3,0)$ \\
\hline
\end{tabular}

SC: Sin complicaciones; CC: Con complicaciones. ${ }^{1}$ Cateterismo de arteria pulmonar, Drogas vasoactivas.

Tabla 3. Razón de prevalencias del tipo de procedimiento utilizado en la instalación de CVC

\begin{tabular}{|c|c|c|c|c|}
\hline & Presencia de co & licaciones & Razón de & IC $95 \%$ \\
\hline Procedimiento ${ }^{1}$ & 0,7 & 18,0 & 0,041 & $0,006-0,299$ \\
\hline
\end{tabular}

`Se consideró como expuesto al grupo de pacientes que recibió la instalación de CVC guiada por ultrasonografía.

convencional, la prevalencia de complicaciones fue de $18 \%$.

Del total de complicaciones, la mayor proporción corresponde a la de procedimientos de urgencia utilizando la técnica convencional $(63,2 \%)$.

En relación al número de punciones realizadas, $85,7 \%$ tuvo éxito en un primer intento, $10,5 \%$ en dos, $2,5 \%$ en tres y $1,3 \%$ en cuatro. Sumado a lo anterior, la mayoría de las complicaciones vinculadas al número punciones $(83,3 \%)$ se produjeron al realizar 2 o más punciones, en el grupo que recibió el procedimiento convencional.

Finalmente, se determinó que la prevalencia de presentar complicaciones era menor en aquellas personas cuya instalación de CVC fue realizada con la ayuda de US (RP $=0,041$; IC 95\% $=0,006$ 0,299 ).

\section{Discusión}

Este primer reporte de la experiencia en Chile en la instalación de vías venosas centrales bajo visión ultrasonográfica está en línea con resultados internacionales, respecto de una menor prevalencia de complicaciones en comparación con la técnica convencional. Ambos grupos resultaron comparables, a excepción de la programación del procedimiento, siendo predominantemente electiva en el grupo de US y de urgencia en el grupo de referencias anatómicas. Sin embargo, en los pacientes del grupo de US que se sometieron a un procedimiento de urgencia, no se contaron complicaciones.

Como en otros estudios ${ }^{14}$ el sitio de punción preferido fue el yugular. La punción arterial fue la única complicación del grupo de US (1 caso) y la 
mayoritaria en el grupo de técnica convencional, lo cual concuerda con resultados de otras series ${ }^{15-18}$.

$\mathrm{Al}$ igual que lo reportado en la literatura ${ }^{17,19}$, en todos los casos incluidos fue posible realizar la instalación exitosa del CVC. Respecto del número de punciones requeridas para una instalación exitosa, nuestro estudio muestra que la técnica basada en US presenta una mayor proporción de uno o dos intentos comparada con el método convencional, como también lo han reportado otros grupos ${ }^{15-17}$.

Entre las limitaciones de este estudio destaca su carácter descriptivo y las dificultades asociadas a la utilización de bases secundarias de datos, por ejemplo, la falta de control de los investigadores sobre aquellos potenciales sesgos asociados a la obtención y registros de los datos, la extención de los periodos de tiempo en que se registraron los mismo, etc. Por otra parte, también cabe señalar que en este estudio en uno de los grupos una de las intervenciones fue realizada por médicos especialistas entrenados en punciones bajo guía ultrasonográfica en modalidad de visualización dinámica. Esta condición podría limitar la validez externa de los resultados. No obstante, lo anterior, y por el carácter y alcance del estudio, solo se pretendió describir dos realidades a nivel de tendencia.

Recomendaciones internacionales refuerzan la necesidad de incorporar el uso de la US en la instalación de CVC para mejorar la seguridad del procedimiento ${ }^{20,21}$, lo cual requiere de un entrenamiento adecuado. En este sentido, y ampliando la utilidad práctica de la US, consideramos que esta debiese formar parte del currículum de formación de un médico en Chile, de manera de entregarle más herramientas para optimizar una hipótesis diagnóstica, al complementar y extender el examen físico ${ }^{9}$, más allá de la demostrada utilidad en diversos procedimientos diagnósticos/ terapéuticos más propios de los postítulos. En esa línea, creemos sería relevante investigar el impacto de la implementación de un programa de entrenamiento en US básica en el pregrado de Medicina en Chile.

Agradecimientos: Agradecemos a los Drs. Enrique Quintana Meneses y Fernando Osorio Gonnet, quienes nos motivaron a integrar la US en los procedimientos invasivos de nuestra área y guiaron con gran generosidad y paciencia nuestros primeros pasos en el aprendizaje de esta técnica.

\section{Referencias}

1. Hind D, Calvert N, McWilliams R, Davidson A, Paisley $\mathrm{S}$, Beverley C, et al. Ultrasonic locating devices for central venous cannulation: meta-analysis. BMJ 2003; 327 (7411): 361.

2. Lamperti $M$, Bodenham AR, Pittiruti M, Blaivas M, Augoustides JG, Elbarbary M, et al. International evidence-based recommendations on ultrasound-guided vascular access. Intensive Care Med 2012; 38 (7): 1105-17.

3. Greitz T. Sven-Ivar Seldinger. American Journal of Neuroradiology 1999; 20 (6): 1180-1.

4. Parienti JJ, Mongardon N, Megarbane B, Mira JP, Kalfon P, Gros A, et al. Intravascular Complications of Central Venous Catheterization by Insertion Site. N Engl J Med 2015; 373 (13): 1220-9.

5. Gibbs FJ, Murphy MC. Ultrasound guidance for central venous catheter placement. Hospital Physician 2006; 42 (3): 23.

6. Callum K, Whimster F. Interventional Vascular Radiology and Interventional Neurovascular Radiology: A Report of the National Confidential Enquiry Into Perioperative Deaths; Data Collection Period 1 April 1998 to 31 March 1999: National Confidential Enquiry into Perioperative Deaths; 2000.

7. Feller-Kopman D. Ultrasound-guided internal jugular access: a proposed standardized approach and implications for training and practice. CHEST Journal 2007; 132 (1): 302-9.

8. Kumar A, Chuan A. Ultrasound guided vascular access: efficacy and safety. Best Practice \& Research Clinical Anaesthesiology 2009; 23 (3): 299-311.

9. Moore CL, Copel JA. Point-of-care ultrasonography. N Engl J Med 2011; 364 (8): 749-57.

10. Shojania KG, Duncan BW, McDonald K, Wachter R, Markowitz A. Making health care safer: a critical analysis of patient safety practices. Evid Rep Technol Assess (Summ) 2001; 43(1): 668.

11. Shekelle PG, Wachter RM, Pronovost PJ, Schoelles K, McDonald KM, Dy SM, et al. Making health care safer II: an updated critical analysis of the evidence for patient safety practices. Evid Rep Technol Assess (Full Rep) 2013; (211): 1-945.

12. Brass P, Hellmich M, Kolodziej L, Schick G, Smith AF. Ultrasound guidance versus anatomical landmarks for internal jugular vein catheterization. Cochrane Database Syst Rev 2015; 1: CD006962.

13. Turker G, Kaya FN, Gurbet A, Aksu H, Erdogan C, Atlas A. Internal jugular vein cannulation: an ultrasound-guided technique versus a landmark-guided technique. Clinics (Sao Paulo, Brazil) 2009; 64(10): 989-92. 
14. Airapetian N, Maizel J, Langelle F, Modeliar SS, Karakitsos D, Dupont H, et al. Ultrasound-guided central venous cannulation is superior to quick-look ultrasound and landmark methods among inexperienced operators: a prospective randomized study. Intensive Care Med 2013; 39 (11): 1938-44.

15. Maecken T, Heite L, Wolf B, Zahn PK, Litz RJ. Ultrasound-guided catheterisation of the subclavian vein: freehand vs needle-guided technique. Anaesthesia 2015; 70 (11): 1242-9.

16. Prabhu MV, Juneja D, Gopal PB, Sathyanarayanan M, Subhramanyam S, Gandhe S, et al. Ultrasound-guided femoral dialysis access placement: a single-center randomized trial. Clinical journal of the American Society of Nephrology: CJASN 2010; 5(2): 235-9.

17. Akoglu H, Piskinpasa S, Yenigun EC, Ozturk R, Dede F, Odabas AR. Real-time ultrasound guided placement of temporary internal jugular vein catheters: Assessment of technical success and complication rates in nephrology practice. Nephrology 2012; 17(7): 603-6.

18. Koroglu M, Demir M, Koroglu BK, Sezer MT, Akhan O,
Yildiz H, et al. Percutaneous placement of central venous catheters: comparing the anatomical landmark method with the radiologically guided technique for central venous catheterization through the internal jugular vein in emergent hemodialysis patients. Acta radiologica (Stockholm, Sweden: 1987) 2006; 47(1): 43-7.

19. Ray BR, Mohan VK, Kashyap L, Shende D, Darlong VM, Pandey RK. Interna jugular vein cannulation: A comparison of three techniques. J Anaesthesiol Clin Pharmacol 2013; 29(3): 367-71.

20. Troianos CA, Hartman GS, Glas KE, Skubas NJ, Eberhardt RT, Walker JD, et al. Guidelines for performing ultrasound guided vascular cannulation: recommendations of the American Society of Echocardiography and the Society of Cardiovascular Anesthesiologists. Anesth Analg 2011; 24 (12): 1291-318.

21. Rupp SM, Apfelbaum JL, Blitt C, Caplan RA, Connis RT, Domino KB, et al. Practice guidelines for central venous access: a report by the American Society of Anesthesiologists Task Force on Central Venous Access. Anesthesiology. [Practice Guideline] 2012; 116 (3): 539-73. 